\title{
Survival of Heparins, Oral Anticoagulants, and Aspirin after the Year 2010
}

\author{
Jawed Fareed, Ph.D., D.Sc., ${ }^{1}$ Debra A. Hoppensteadt, Ph.D., ${ }^{1}$ Daniel Fareed, ${ }^{2}$ \\ Muzaffer Demir, M.D., ${ }^{2}$ Rakesh Wahi, M.D., 3 Melaine Clarke, ${ }^{1}$ \\ Cafer Adiguzel, M.D., 1 and Rodger Bick, M.D., Ph.D., F.A.C.P. 4
}

The conventional management of thrombotic and cardiovascular disorders is based on the use of heparin, oral anticoagulants, and aspirin. Despite remarkable progress in life sciences, these drugs still remain a challenge and a mystery to us, and their use is far from optimized. The development of low-molecular-weight heparins and the synthesis of heparinomimetics, such as the chemically synthesized pentasaccharide, represent a refined use of heparin. Additional drugs from this knowledge will continue to develop; however, none of these drugs will ever match the polypharmacology of heparin. Aspirin still remains the leading drug in the management of thrombotic and cardiovascular disorders. The newer antiplatelet drugs such as adenosine diphosphate receptor inhibitors, glycoprotein IIb/IIIa inhibitors, and other specific inhibitors have limited effects and have been tested in patients who have already been treated with aspirin. Warfarin provides a convenient and affordable approach in the long-term outpatient management of thrombotic disorders. The optimized use of these drugs still remains as the approach of choice to manage thrombotic disorders. The new anticoagulant targets, including specific sites in the hemostatic network such as tissue factor, individual clotting factors (IIa, VIIa, IXa, Xa, XIIa, and XIIIa), recombinant forms of serpins (antithrombin, heparin cofactor II, and tissue factor pathway inhibitors), recombinant activated protein $\mathrm{C}$, thrombomodulin, and site-specific serine protease inhibitor complexes have also been developed. There is a major thrust on the development of orally bioavailable anticoagulant drugs (anti-Xa and anti-IIa agents), which are slated to replace oral anticoagulants. Both the anti-factor Xa and antithrombin agents have been developed for oral use and have provided impressive clinical outcomes in sponsor trials for the postsurgical prophylaxis of venous thrombosis; however, safety concerns related to liver enzyme elevations and thrombosis rebound have been reported with their use. For these reasons, the U.S. Food and Drug Administration did not approve the orally active antithrombin agent ximelagatran for several indications. The synthetic pentasaccharide (fondaparinux) has undergone an aggressive clinical development. Unexpectedly, fondaparinux also produced major bleeding problems at minimal dosages. Fondaparinux represents only one of the multiple pharmacologic effects of heparins. Thus, its therapeutic index will be proportionately narrower. The newer antiplatelet drugs have

${ }^{1}$ Hemostasis and Thrombosis Research Laboratories, Loyola University Medical Center, Maywood, Illinois; ${ }^{2}$ Trakya University School of Medicine, Department of Medicine, Division of Hematology, Edirne, Turkey; ${ }^{3}$ Grant Memorial Hospital, Petersburg, West Virginia; ${ }^{4}$ University of Texas Southwestern Medical Center, Dallas, Texas.

Address for correspondence and reprint requests: Jawed Fareed, Ph.D., Professor of Pathology and Pharmacology, Director, Hemostasis and Thrombosis Research Laboratories, Loyola University
Medical Center, 2160 S. First Avenue, Maywood, IL 60153 (e-mail: jfareed@lumc.edu).

Hot Topics II: An Editorial Collection of Current Issues and Controversies in Thrombosis and Hemostasis; Guest Editor, Emmanuel J. Favaloro, Ph.D., M.A.I.M.S.

Semin Thromb Hemost 2008;34:58-73. Copyright (C) 2008 by Thieme Medical Publishers, Inc., 333 Seventh Avenue, New York, NY 10001, USA. Tel: +1(212) 584-4662.

DOI 10.1055/s-2008-1066025. ISSN 0094-6176. 
added a new dimension in the management of thrombotic disorders. The favorable clinical outcomes with aspirin and clopidogrel have validated cyclooxygenase (COX)-1 and P2Y 12 receptors as targets for new drug development. Prasugrel, a novel thienopyridine, cangrelor, and AZD 6140 represent newer P2Y 12 antagonists. Cangrelor and AZD 6140 are direct inhibitors, whereas prasugrel requires metabolic activation. Though clinically effective, recent results have prompted a closure of a large clinical trial with prasugrel due to bleeding. The newer anticoagulant and antiplatelet drugs are attractive for several reasons; however, none of these are expected to replace the conventional drugs in polytherapeutic approaches. Heparins, warfarin, and aspirin will continue to play a major role in the management of thrombotic and cardiovascular disorders beyond 2010.

KEYWORDS: Heparin, warfarin, aspirin, antithrombins, anti-Xa agents

Over the past decade, interest in anticoagulant drugs has grown dramatically, as evidenced by a continual increase in the number of drugs introduced for both preclinical and clinical development. ${ }^{1,2}$ These drugs include the new heparins, synthetic heparinomimetic agents, antithrombin agents, anti-Xa agents, biotechnology-derived antithrombotic proteins, and newer antiplatelet drugs. The newer drugs represent a wide array of chemicals and biologics with both structural and functional diversity (Fig. 1). These drugs represent proteins, carbohydrates, synthetic organomimetics, and biotechnology-derived agents. The outstanding scientific research and development activities in the academic centers and pharmaceutical industry have resulted in a steady flow of many of the new products including the following:

1. Ultralow-molecular-weight heparins (bemiparin, deligoparin, and octaparin).

2. Heparinomimetics such as fondaparinux and idraparinux.

3. Synthetic direct antithrombin agents (parenteral and oral).

4. Synthetic direct anti-Xa agents (parenteral and oral).

Of these agents, fondaparinux is in advanced clinical development for various indications including postsurgical deep vein thrombosis (DVT) and acute coronary syndrome (ACS). The parenteral antithrombin agents are primarily used for substitute anticoagulation for heparin-compromised patients (i.e., heparin-induced thrombocytopenia syndrome; HITS). The oral anti-Xa and anti-IIa agents are currently developed for the potential replacement of warfarin and heparins; however, safety considerations and rebound thrombosis are major concerns. Thrombosis is a polypathologic syndrome, where blood and endothelial cells, plasmatic components, inflammatory responses, and hemodynamic abnormalities contribute to its pathogenesis (Fig. 2). Single-target drugs such as the newly developed antiprotease agents may have a limited value in the management of throm- bosis and only in combined modalities; these agents may mimic polytherapeutic actions of conventional drugs.

Extensive clinical trials have been performed globally to support the claims of the safety and efficacy of the newer drugs. Several reports on the possible replacement of warfarin by newly developed oral antithrombin agents have become available. ${ }^{3,4}$ Through their fast track and revised policies, the regulatory bodies such as the European Medicine Evaluation Agency (EMEA), U.S. Food and Drug Administration (FDA), and other regional agencies have continually contributed to the timely evaluation and approval of new drugs by providing input at various stages of drug development. Such close interactions have clarified various issues related to drug development and in fact have accelerated the approval process of many new drugs such as low-molecular-weight heparins (LMWHs), synthetic heparin pentasaccharide (fondaparinux), and activated protein $\mathrm{C}$ (Xigris [drotrecogin alfa], Eli Lilly, Indianapolis, IN).

Owing to the dramatic development and the relatively defined chemical and biologic profile of the newer drugs, it is now widely perceived that the conventional anticoagulants such as the heparins, warfarin, and aspirin will eventually be replaced by newer drugs. ${ }^{4}$ This is partly due to several reported problems with their use and associated adverse reactions. Table 1 lists the conventional drugs and their potential substitutes for various indications. Unfractionated heparin (UFH) has been in use for nearly 50 years. It is the only anticoagulant drug with an antidote. In many countries, this anticoagulant still remains the main drug for anticoagulant management of thrombosis and cardiovascular disorders. Warfarin still remains the drug of choice for the outpatient management of thrombosis, whereas aspirin has been used for multiple indications for a long period of time.

The use of UFH has been optimized by developing the LMWHs. Therefore, the LMWHs actually represent an optimized use of heparin. This is mainly due to our current understanding of the chemistry and biology of heparin. Antithrombin drugs such as lepirudin, argatroban, and bivalirudin have been in development for 


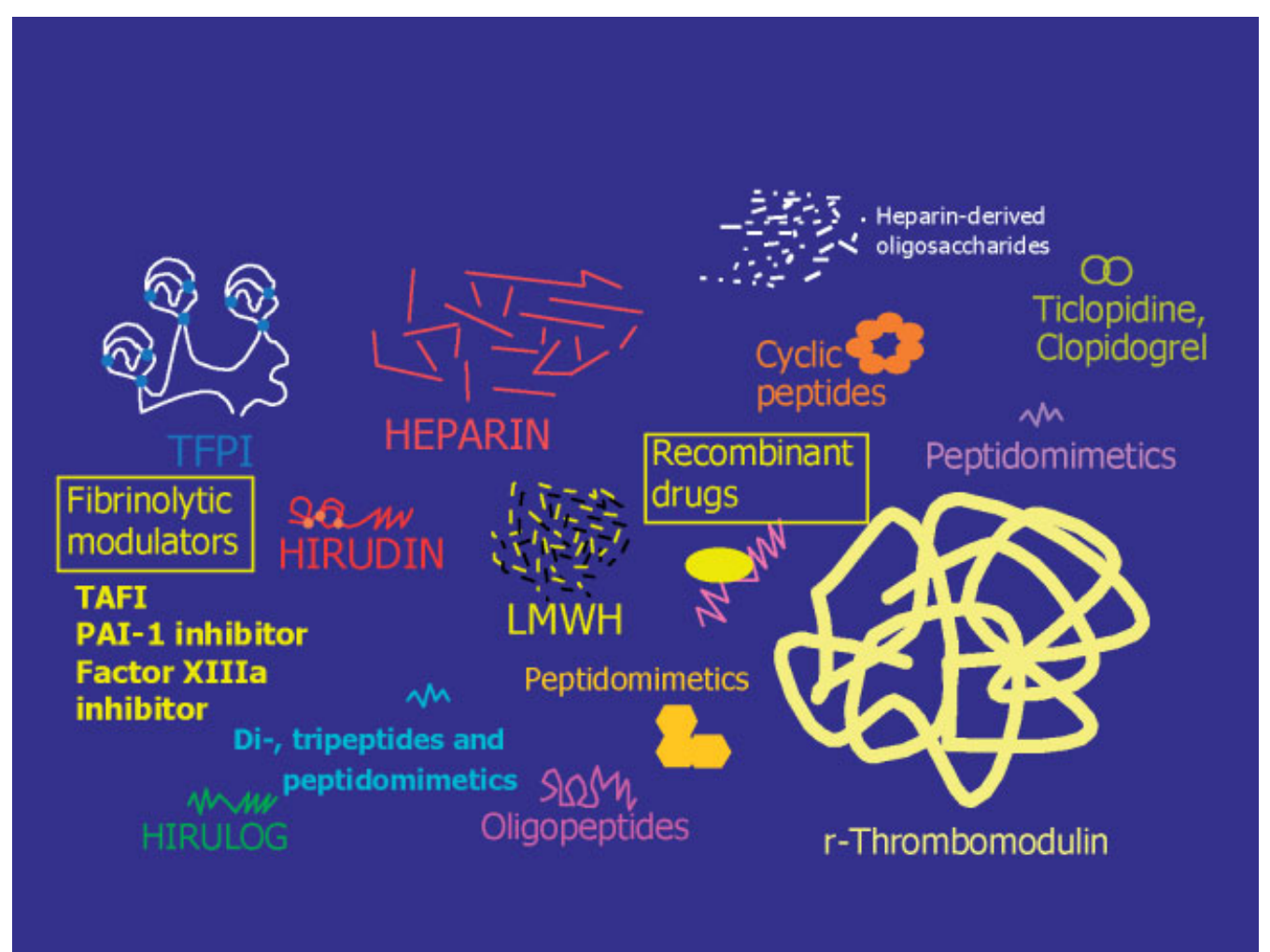

Figure 1 The newly developed anticoagulant drugs represent a variety of synthetic, biologic, hybrid, and biotechnology-derived drugs. These drugs exhibit wide structural and functional heterogeneity. The heparin-derived drugs include high-potency unfractionated heparin (UFH), low-molecular-weight heparins (LMWHs) and their generic equivalents, ultralow molecular weight heparins and heparin-derived oligosaccharides. The synthetic heparinomimetics include fondaparinux and idraparinux. The specific anti-Xa and anti-Ila drugs are organomimetics and directly inhibit factor Xa and thrombin, respectively. The biotechnology-derived drugs include recombinant protein equivalents of lepirudin, thrombomodulin, activated protein $\mathrm{C}$, and antithrombin. Newer antiplatelet drugs include clopidogrel and other ADP receptor inhibitors such as crangelor and prasugrel. TFPI, tissue factor pathway inhibitor; TAFI, thrombin-activatable fibrinolysis inhibitor; PAI-1, plasminogen activator inhibitor-1.

many years. These drugs are useful as a substitute for heparin in such conditions as heparin-induced thrombocytopenia (HIT); however, these drugs do not have any antidote and cannot be used for surgical indications at this time. The anti-Xa drugs and the heparinomimetics do not have any direct effect on thrombin and produce minimal anticoagulant effects; therefore, these drugs may or may not be useful in the management of patients who are heparin compromised. However, the long-term use of these agents requires further clinical validation. Also, whereas heparin represents a polytherapeutic drug, the newer drugs tend to be monotherapeutic. Thus, their indications will be somewhat limited. Moreover, some of the therapeutic effects observed with heparins may not be seen with the newer drugs.

It is more than 40 years since the oral anticoagulant drugs such as warfarin began to be used for the management of thrombotic and cardiovascular disorders. Response variation, need for monitoring, and delayed onset/cessation are some of the problems associated with its continued use. More recently, the oral antithrombin drugs, such as ximelagatran, have been developed as potential substitutes for warfarin. ${ }^{3}$ Though this agent is shown to be effective and in some cases noninferior to warfarin, its use has been associated with an increase in liver enzymes, and this agent passes the placental and blood-brain barriers. For these reasons, the FDA has disapproved the clinical use of ximelagatran in various indications. This drug was also withdrawn from the European countries by AstraZeneca (Wilmington, DE) due to safety concerns. It development and clinical use is now completely stopped.

Several oral anti-Xa agents are also in clinical development at this time. ${ }^{5}$ Unlike the antithrombin agents, these drugs do not compromise the regulatory function of thrombin. Furthermore, it is believed that these agents may have a broader therapeutic index than that of the oral thrombin inhibitors. Because the target is factor $\mathrm{Xa}$, these drugs may mimic oral anticoagulants in pharmacodynamic actions. Initial studies on various factor $\mathrm{Xa}$ inhibitors have had promising results; however, additional trials by directly comparing these agents with oral anticoagulants are needed. The development of oral heparins and related drugs has been somewhat disappointing as these agents were not as effective as the subcutaneous LMWHs. A direct comparison 


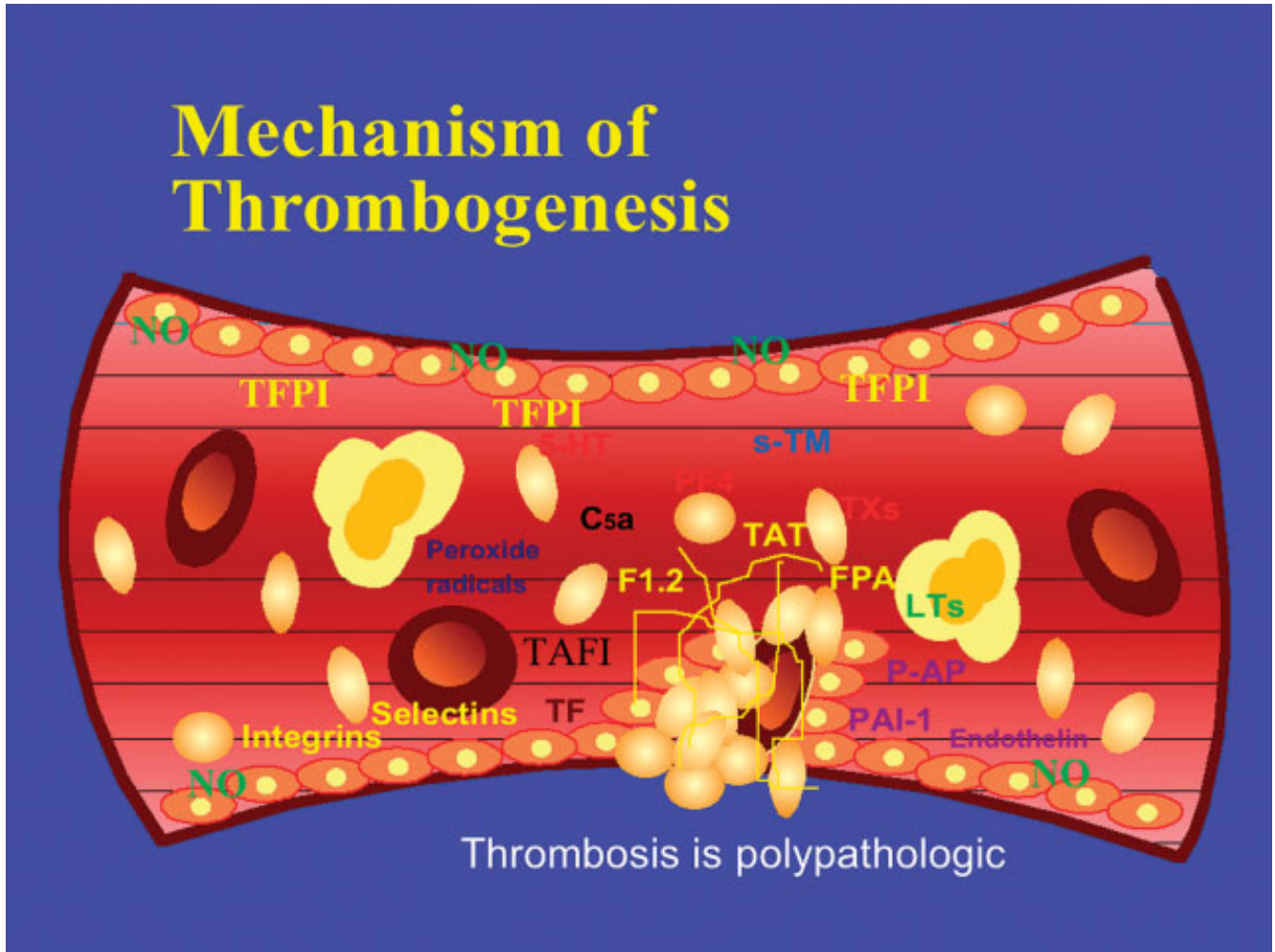

Figure 2 Thrombogenesis is a complex multicomponent pathologic process involving both cellular and humoral factors. Endothelial damage, cellular activation, release of mediators (including inflammatory components), and modulators of vascular tone all contribute to the overall pathogenic mechanisms. The complete understanding of the thrombogenic process is not known, but it is known that it is not fully dependent on the generation of thrombin and related enzymes. TFPI, tissue factor pathway inhibitor; TAFI, thrombin-activatable fibrinolysis inhibitor; PAl-1, plasminogen activator inhibitor-1; TF, tissue factor; $\mathrm{NO}$, nitric oxide; $\mathrm{TXB}_{2}$, thromboxane $\mathrm{B}_{2}$; LTs, leukotriene; FPA, fibrinopeptide A; PAl-1, plasminogen activator inhibitor-1; PF4, platelet factor 4; C5a, complement C5a; s-TM, soluble thrombomodulin; F1.2, prothrombin fragment F1.2, PAP, plasminogen antiplasmin complex; TAT, thrombin antithrombin complex.

between the anti-Xa agents and oral anticoagulant drugs is not available at this time. Thus, additional clinical trials are needed to further validate the therapeutic index of these agents in comparison with warfarin.

Table 1 Conventional Anticoagulants and Antithrombotic Drugs and Their Potential Substitutes

\begin{tabular}{ll}
\hline Conventional Drug & Potential Substitute \\
\hline Unfractionated heparin & $\begin{array}{c}\text { LMWHs, antithrombin } \\
\text { agents, anti-Xa agents, } \\
\text { heparinomimetics } \\
\text { (fondaparinux) }\end{array}$ \\
& Oral antithrombin drugs \\
Oral anticoagulant drugs & Oral anti-Xa drugs \\
(warfarin and related drugs) & Oral heparinomimetics \\
Antiplatelet agents & Selective COX inhibitors \\
& Aspirin \\
& ADP receptor inhibitors \\
& Phosphodiesterase inhibitors \\
& GP Ilb/Illa inhibitors \\
\hline
\end{tabular}

LMWH, low molecular weight heparin; COX, cyclooxygenase receptor; ADP, adenosine diphosphate; GP Ilb/Illa, glycoprotein IIb/lla.
Aspirin has been in clinical use for more than 100 years. The antiplatelet effect of this agent was recognized some 40 years ago. Since then, aspirin has been a lifesaving drug for several types of thrombotic indications. Several newer formulations of aspirin have been developed. Aspirin now represents a universal antithrombotic drug in both thrombotic and cardiovascular indications. The newly developed cyclooxygenase (COX) inhibitors exhibit some specific effects of aspirin but may or may not exhibit the potential therapeutic effects in thrombosis. As a matter of fact, due to the specificity of these agents, they may exhibit thrombotic complications. Table 2 shows a comparison between

\section{Table 2 Aspirin Versus ADP Receptor Inhibitors}

\begin{tabular}{lc}
\hline Aspirin & ADP Receptor Inhibitor \\
\hline Polypharmacologic effects & Single receptor targeting agent \\
Produces both platelet and & Only produces platelet \\
vascular effects & mediated responses \\
$\begin{array}{c}\text { Multiple actions (analgesic, } \\
\text { anti-inflammatory) }\end{array}$ & Only produces inhibition \\
\hline
\end{tabular}

ADP, adenosine diphosphate. 
aspirin and adenosine diphosphate (ADP) receptor inhibitors. In comparison with aspirin, the ADP receptor inhibitors are single-target drugs. The selective ADP receptor inhibitors, when combined with aspirin, exhibit superior efficacy in comparison with monotherapy. However, their clinical spectrum without aspirin will be limited. Thus, it is highly unlikely that the newer antiplatelet drugs will provide the broad therapeutic index observed with aspirin, including the anti-inflammatory and immunomodulatory actions.

Owing to rapid developments in these potential therapeutics, several important issues related to current practices in anticoagulant therapy should be recognized. These issues include:

1. The replacement of UFH by LMWHs in all indications including medical and surgical anticoagulation.

2. The potential replacement of heparins by newly developed antithrombin and anti-Xa agents.

3. The feasibility of oral anti-Xa and anti-IIa agents as potential substitutes for oral anticoagulant drugs.

4. The development of synthetic heparinomimetics representing specific actions of heparins and their relative bioequivalence to heparin.

5. The development of recombinant antithrombotic agents such as activated protein C (APC), tissue factor pathway inhibitor, recombinant equivalent of serpins, and thrombomodulin, with reference to their relative applications in specific disorders.

6. The development of newer antiplatelet drugs such as the ADP receptor inhibitors, glycoprotein (GP) IIb/ IIIa receptors, phosphodiesterase inhibitors, and specific COX-1 and COX-2 inhibitors and their relevance in the management of vascular disorders. The relevance of on-board aspirin for the therapeutic index of each of these agents also requires additional investigation. COX-2 inhibitors have been known to produce hypercoagulable states due to the sparing of COX-1.

7. The design of newer thrombolytic agents, with specific reference to their endogenous interaction and pharmacodynamic differences in terms of their relative clinical effects in stroke and myocardial infarction.

8. The recent recognition of the antithrombotic actions of statins, nitric oxide donors, and other non-anticoagulant drugs and their impact on overall therapeutic approaches.

9. Because the patents for some of the anticoagulant drugs such as the LMWHs are due to expire, it is likely that generic versions of some of these agents, including enoxaparin and dalteparin, may soon become available. ${ }^{6}$ However, if the generic drugs are approved based solely on the current specifications/ guidelines, many products with similar characteristics but nonsimilar biological or clinical equivalence will be introduced. This may result in safety and efficacy compromise. The need for systematic review and newer guidelines therefore exists at all levels.

Despite several limitations, heparin is still the most widely used anticoagulant in the world. The clinical experience with heparin and the fundamental understanding of its biologic actions have provided additional insights for optimizing the use of this drug. These include the development of heparin fractions, improved production methods to remove viral contaminants and other impurities, understanding of the structure of heparin and development of heparin derivatives, and better understanding of the direct and indirect mechanisms of action. This has led to improved clinical outcomes and reduction of adverse event incidence, such as bleeding and HIT.

\section{LMWHs AND RELATED DRUGS}

Though heparin remains the sole anticoagulant used for interventional surgical procedures, the continual expansion of the newer applications of $\mathrm{LMWHs}$ has added a new dimension to the overall management of thrombotic and cardiovascular disorders. Evidently, the LMWHs have achieved gold standard status in the management of thromboembolic disorders and now challenge other treatments, such as oral anticoagulants, for various indications. Several recent clinical trials have provided supportive data for the polytherapeutic use of LMWHs in the management of coronary syndromes, thrombotic stroke, and malignancy-associated thrombotic events. LMWHs have also shown efficacy as surgical and interventional anticoagulants. ${ }^{7}$ Unlike heparin, these drugs exhibit a better therapeutic index in these indications. LMWHs have also recently been evaluated in atrial fibrillation and cardiac transplantation. These drugs represent a refined use of heparins. Being polypharmacologic in nature, the LMWHs have multiple sites of action. Their actions are not only limited to the inhibition of coagulation enzymes, but these drugs also exhibit profound actions on endothelial sites and blood cells. This has led to the development of the newer forms of LMWHs with structural and functional modifications.

Antithrombin agents such as lepirudin and bivalirudin also have been compared with LMWHs for postsurgical prophylaxis of thromboembolism. Initial reports indicate favorable results with the use of recombinant lepirudin for treatment of coronary syndromes. However, safety issues such as bleeding remain a concern. Understanding the mechanisms of antithrombotic actions and the relevance of structural components of LMWHs has led to the development of synthetic analogues of heparin fragments. One approach, based on the elucidation of the structure of heparin, has led to the synthesis of oligosaccharides with high affinity for antithrombin (AT). Whereas synthetic fondaparinux 
has undergone extensive clinical trials for both thromboembolic and coronary indications, safety considerations such as bleeding and catheter thrombosis remain. It is important to point out that fondaparinux exhibits monotherapeutic effects, which are mainly mediated via the inhibition of factor Xa. Although the development of the synthetic pentasaccharides represents a major advance in producing heparin-like drugs using synthetic organic methods, this agent produces only a single pharmacologic action of heparin. Furthermore, the pharmacologic actions of these oligosaccharides are dependent on endogenous antithrombin. The FDA and the EMEA have approved the use of synthetic heparin pentasaccharide, fondaparinux, for the management of post-orthopedic surgical thrombosis. However, bleeding risk was unexpectedly higher with this drug, and its use is not recommended in underweight patients. Fondaparinux is likely to be equivalent to other modalities in the management of DVT prophylaxis; however, its use in other indications where LMWHs are approved may not provide equivalence or superiority due to its monotherapeutic nature.

Several additional clinical trials are being performed on fondaparinux in multiple indications, including treatment of thrombosis. Besides the lack of a clear clinical response, bleeding issues, nonavailability of an antidote, drug interactions, product accumulation, and thrombocytopenia are some of the issues that will require clarification. The current clinical trials may provide some of the answers on these issues. Heparin and LMWHs are polycomponent drugs with multiple actions. Furthermore, these drugs also release tissue factor pathway inhibitor from endogenous sites. Thus, these agents may have a relatively broader therapeutic index.

Clinical trials in Europe have shown that subcutaneous LMWHs, given once or twice daily, are at least as safe and effective as continuous intravenous heparin in the prevention of recurrent venous thromboembolism and are associated with reduced bleeding and lower mortality rates. Several recent studies have shown that home administration of $\mathrm{LMWH}$ s is as safe and effective as hospital administration of intravenous heparin in patients with proximal vein thrombosis. Initial evidence clearly suggests the LMWHs may be a useful alternative to heparin in patients with pulmonary embolism. LMWHs also may be useful alternatives to heparin for arterial indications, such as treatment of unstable angina and stroke and the maintenance of peripheral arterial grafts.

Recognizing the usefulness of LMWHs, the pharmaceutical industry has focused its attention on their use in the management of ischemic and thrombotic stroke. The success of early clinical trials also suggests that LMWHs may be useful in the management of primary and secondary ischemic or thrombotic stroke. Though in several clinical trials the LMWHs did not show any improvement for the neurovascular deficit in stroke, these drugs showed a clear reduction in the incidence of thrombotic complications in stroke patients. Thus, in the near future, the use of LMWHs for prevention of thrombotic or ischemic stroke will be an important goal. LMWHs have also shown efficacy in vascular dementia of Alzheimer's type (SDAT). Thus, these drugs may become useful in neurologic disorders. There are several studies that are currently ongoing on the effect of LMWHs and related drugs in neurodegenerative diseases.

Although LMWHs are proving to be as effective as, or safer than, heparin for various indications, it is important to realize that the differences in the manufacturing of various LMWHs may lead to differences in the pharmacologic profile. Although these differences have not been clinically validated, each of the LMWHs is expected to exhibit its own therapeutic index in a given clinical setting. Thus, unlike heparin, the interchanging of LMWHs based on equivalent gravimetric or biologic potency of standardized dosages may not be feasible. Optimized dosages of various LMWHs have been established for prophylaxis and treatment of DVT. Thus, each agent is given at a specified dosage. The optimized dosage of different LMWHs also differs for the management of ACS. The most notable differences are observed at higher dosages. When these agents are given intravenously for interventional cardiovascular procedures, each of the LMWHs produces a different anticoagulant response regardless of the dosage equivalence at the gravimetric or bioassay adjusted potency. Therefore, the FDA, WHO, and professional organizations consider each drug distinct. Because of the newer indications and length of therapy, some additional issues related to the optimal use of LMWHs remain to be addressed. Examples include monitoring, control of bleeding, and drug interactions. In addition, the use of high-dose subcutaneous LMWHs may require pharmacologic antagonism. Several clinical trials have been designed to obtain information related to these issues. The differential clinical efficacy of various LMWHs was evident in the trials performed with dalteparin (FRISC and FRIC), enoxaparin (ESSENCE), and nadroparin (FRAXIS). ${ }^{8}$

The LMWHs have also shown remarkable clinical efficacy in the management of cancer-associated thrombosis. ${ }^{9}$ More recently, the FDA has approved a LMWH, namely dalteparin, for the management of cancer-associated thrombosis. Additional clinical trials have shown that LMWHs reduce the mortality in cancer patients. Several clinical studies in medical patients for the prophylaxis and treatment of thrombosis have provided supportive data that cancer-associated mortality can be reduced by using LMWHs. Thus, besides the anticoagulant effects, there may be additional actions of these agents, which warrants further investigation. 
Additional depolymerization of LMWHs has resulted in the development of ultralow-molecularweight heparins. Several of these have recently become available. Bemiparin represents such a product that has been found efficacious in the management of DVT in Europe. Several other agents are being clinically tested in several possible indications including vascular dementia, inflammatory bowel disease, and ACS. Many of the major pharmaceutical companies are currently developing various forms of LMWHs with averaged molecular weight $<3000$ Da. These lowered-molecular-weight heparins may exhibit better safety and efficacy indices.

\section{DIRECT THROMBIN INHIBITORS}

Understanding the coagulation process has led to the identification of thrombin as a key enzyme in the thrombogenic processes. Several direct thrombin inhibitors have been developed over the past few years. ${ }^{10}$ Argatroban, bivalirudin, and lepirudin have become available for alternative management of patients who are refractory to heparin. The recognition of HIT as the most catastrophic adverse effect of heparin has led to the use of alternate anticoagulants. The antithrombin agents are most useful in this indication and have been specifically developed for HIT. Lepirudin, the leech-derived protein, has been compared with heparin for various indications, including treatment and prophylaxis of venous and arterial thrombotic disorders. The use of lepirudin has been reported to be associated with increased risk of bleeding, indicating that better monitoring and dose-adjustment protocols are needed as well as antidotes. To date, clinical trials comparing lepirudin and heparin as adjuncts in thrombolytic therapy in myocardial infarction (TIMI 9B) and acute coronary syndromes (GUSTO IIb) have shown lepirudin to be marginally (if at all) superior to heparin. ${ }^{11,12}$ Recently, several reports comparing the effects of heparin and lepirudin have become available. A study comparing heparin and recombinant lepirudin for the prophylaxis of DVT provided impressive data in favor of lepirudin. ${ }^{13}$ In a second study, LMWHs also were compared with lepirudin for postsurgical prophylaxis of DVT. ${ }^{14}$ The results favored lepirudin. Both studies emphasize an important point about the validity of well-designed clinical trials. It is important to understand that the efficacy and safety of a new drug may not be determined by trials for a single indication. The use of lepirudin is associated with the generation of antibodies, which can impair the pharmacokinetics and pharmacodynamics of this drug. Severe anaphylactic reactions have also been reported with the use of lepirudin. Therefore, additional clinical trials are needed to demonstrate the safety of lepirudin and related drugs.

Bivalirudin represents a designer antithrombin drug that combines the features of lepirudin and other anticoagulant peptides. It is a reversible antithrombin agent and offers several advantages over lepirudin. This agent has undergone several clinical trials for interventional and surgical anticoagulation. ${ }^{15-18}$ The FDA has approved this agent for anticoagulation during percutaneous coronary angioplasty (PTCA). Currently, bivalirudin is undergoing clinical trials for cardiovascular bypass surgery. Furthermore, antithrombin agents such as argatroban and bivalirudin may be useful in off-pump bypass surgery. Antibody generation to bivalirudin has also been reported. ${ }^{19}$ This can also influence the dosing and the relative responses of this drug.

Argatroban, a small peptidomimetic thrombin inhibitor, is also approved by the FDA as an alternate anticoagulant for patients with HIT. It has been used successfully in Japan for more than a decade in the treatment of thrombotic disorders. Several clinical trials in both Europe and the United States have been designed to investigate its use as an alternative to heparin in heparin-compromised patients and as a prophylactic agent to reduce late restenosis after PTCA and coronary directional atherectomy (CDA). ${ }^{20}$ Argatroban has been successfully used as an anticoagulant in patients with HIT and as a substitute for heparin in PTCA. ${ }^{21}$ Because the half-life of argatroban is rather short, it has been administered via infusion protocols. For therapeutic anticoagulation, a level of 1 to $2 \mu \mathrm{g} / \mathrm{mL}$ is indicated, whereas for interventional cardiology procedures a level of 3 to $7 \mu \mathrm{g} / \mathrm{mL}$ is necessary. Argatroban also exhibits additional actions on blood vessels and may exert its clinical effects via multiple measures.

\section{DIRECT Xa INHIBITORS}

Because of their weaker anticoagulant effects in global clotting tests, direct factor $\mathrm{Xa}$ inhibitors were not considered desirable anticoagulant and antithrombotic agents for developmental purposes. However, because of favorable clinical results with fondaparinux, strong interest in synthetic anti-factor Xa drugs has reemerged. These agents may be useful in the prophylaxis of both arterial and venous thrombotic disorders and may offer a greater margin of safety than do existing drugs. Additional advantages of direct thrombin and factor $\mathrm{Xa}$ inhibitors over heparin include subcutaneous and oral bioavailability. Although their biologic half-life is usually less than 30 minutes, coupling to larger agents such as dextran or albumin can prolong their half-life without affecting their pharmacologic actions. Questions about monitoring and antagonism will have to be answered before thrombin inhibitors and factor $\mathrm{Xa}$ inhibitors can be widely explored in clinical settings. Depending on their specificity for thrombin or factor Xa, they may be used as adjuncts with other classes of drugs, such as thrombolytic agents for treatment of acute myocardial 


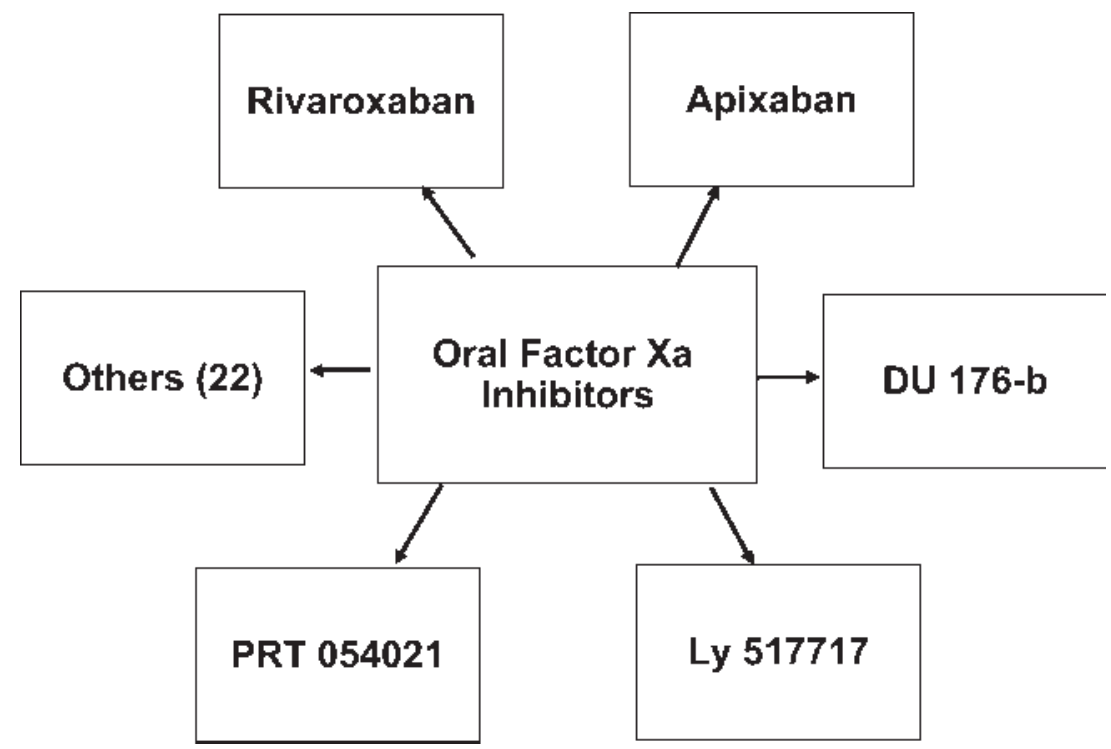

Figure 3 The current development of factor Xa inhibitors represents a major aspect of anticoagulant drug development with these agents in different stages of development. Most of the anti-Xa agents are small-molecules, synthetic compounds that are developed for specific clinical indications. Rivaroxaban (Bayer, Leverkusen, Germany), apixaban (Bristol Meyers-Squibb, New York, NY), DU-176b (Daiichi-Sankyo, Parsippany, NJ), LY-517717 (Lilly, Indianapolis, IN), and PRT-054021 (Portola, San Francisco, CA) represent some of these synthetic agents in different stages of clinical development. There are more than 20 other factor Xa inhibitors, which are in different stages of development. Although these agents are called factor Xa inhibitors, because of their structure, they may have additional pharmacologic actions, which are not fully explored at this time. Unlike heparin, the factor Xa inhibitors are homogeneous, synthetic, and monotherapeutic agents. The anticoagulant activities of these agents may not be directly proportional to the inhibition of factor Xa.

infarction. Low-molecular-weight thrombin inhibitors and factor Xa inhibitors also may be used for localized delivery, stenting, and transdermal delivery.

At the present time, several anti-Xa drugs are currently being developed for various indications (Fig. 3). A major interest in the area is to develop oral anti-Xa drugs that can be used for the long-term management of both the arterial and venous thrombosis. Because of their better bioavailability, thrombin inhibitors and factor $\mathrm{Xa}$ inhibitors in combination may be more useful than the single agents. Optimal combinations for specific indications may be considered. As in the clinical development of LMWHs, thrombin inhibitors and factor Xa inhibitors should be compared with heparin in terms of safety, efficacy, and cost.

Both the oral antithrombin and anti-Xa agents are currently proposed as potential substitutes for oral anticoagulants for the long-term management of thrombotic and cardiovascular indications. Ximelagatran has undergone extensive clinical trials in DVT prophylaxis and atrial fibrillation. ${ }^{22}$ In comparison with LMWH and warfarin, it has exhibited variable safety and efficacy. In the atrial fibrillation trial, it was shown to be noninferior. ${ }^{23}$ Similarly, some recent clinical trials have also reported on the efficacy of oral anti-Xa agents. ${ }^{24,25}$ These agents are in their early clinical development and have some promising initial reports. Though there is discussion that oral anticoagulants such as warfarin can be replaced by oral anti-IIa or anti-Xa agents, the use of warfarin is currently being further optimized. With the use of the international normalized ratio (INR), some global harmonization for its use has been achieved. Self-monitoring is providing a patient-driven control process analogous to the regulation of insulin therapy. Newer formulations have recently become available. New trials have provided useful data on its efficacy. Thus, optimized use of warfarin may turn out to be as safe and effective as the newly developed anti-Xa and IIa agents.

\section{ANTICOAGULANT DRUGS IN THE MANAGEMENT OF CORONARY INTERVENTIONS}

Anticoagulant drugs are crucial in the management of patients with percutaneous coronary intervention (PCI). Anticoagulants used in patients undergoing PCI should not only prevent coronary events but also maintain catheter patency to avoid complications. Beside the use of UFH, newer approaches for PCI include LMWHs and other agents such as fondaparinux and bivalirudin. Lepirudin was initially used in PCI, however, because of safety considerations, its use was not considered optimal. Both UFH and enoxaparin have a good efficacy and safety profile in PCI. In addition, the incidence of procedural complications such as catheter thrombosis is 
a rare event. In contrast, recent clinical trial data have indicated that factor $\mathrm{Xa}$ inhibition with agents such as fondaparinux may be associated with an increased incidence of catheter thrombosis compared with heparin-based anticoagulants (UFH and LMWH). ${ }^{26}$ Experimental systems show that the polytherapeutic agents UFH and enoxaparin are more effective anticoagulants than certain single-target factor Xa inhibitors, such as fondaparinux. ${ }^{27}$ On the other hand, antithrombin agents such as bivalirudin have been found to be more effective anticoagulants in PCI settings. ${ }^{28}$ The safety and efficacy of using the direct thrombin inhibitor, bivalirudin, during PCI was first evaluated in the REPLACE-2 trial, which reported the noninferiority of bivalirudin to heparin for this indication. ${ }^{15}$ In the subsequent Acute Catheterization and Urgent Intervention Triage Strategy (ACUITY) study, patients with moderate- to high-risk ACS were randomized to receive enoxaparin, UFH plus GP IIb/IIIa inhibitors, or bivalirudin with and without GP IIb/IIIa inhibitors. ${ }^{16,18}$ A subanalysis of patients who underwent PCI indicated similar rates of composite ischemic outcomes across all treatment groups. Rates of major bleeding were similar in patients receiving UFH or bivalirudin with GP IIb/IIIa inhibitors; however, the rate of major bleeding was significantly lower in the bivalirudin monotherapy group compared with both UFH and bivalirudin plus GP IIb/IIIa inhibitors. No cases of catheter-related thrombus formation were reported in patients receiving UFH or bivalirudin with or without GP IIb/IIIa inhibitors. Bivalirudin monotherapy may therefore be a promising alternative to UFH therapy in a GP IIb/IIIa inhibitor-sparing strategy.

The larger-scale, recently completed Harmonizing Outcomes with Revascularization and Stents in AMI (HORIZONS AMI) trial compared the use of bivalirudin monotherapy with UFH plus GP IIb/IIIa inhibitors in patients with ST-segment elevation myocardial infarction. ${ }^{17}$ In the subpopulation of patients who underwent primary PCI, bivalirudin monotherapy was associated with a significant $24 \%$ reduction in the risk of net adverse clinical events up to 30 days postrandomization, largely driven by a $40 \%$ reduction in the risk of non-coronary bypass graft (CABG)-related major bleeding. However, the authors also reported a significantly greater incidence of acute stent thrombosis (occurring $\leq 24$ hours after PCI) in patients receiving bivalirudin $(1.3 \%$ vs. $0.3 \%$ in patients receiving bivalirudin and UFH plus GP IIb/IIIa inhibitors, respectively; $p=0.0009)$. The overall incidence of stent thrombosis was similar in the two treatment groups $(2.5 \%$ vs. $1.9 \%$ in patients receiving bivalirudin monotherapy and UFH plus GP IIb/IIIa inhibitors respectively; $p=0.33$ ).

Argatroban represented the very first parenteral antithrombin agent used for various indications in Japan. It was later developed for the alternate anticoagulant management of heparin-compromised patients. ${ }^{20,21}$
This agent has also been used in several clinical trials for anticoagulation in PCI. ${ }^{29}$ However, it is not approved for this indication at this time. In the United States, argatroban is widely used for anticoagulation of patients who develop antibodies to heparins (HIT antibodies).

Novel anticoagulants that directly inhibit factor $\mathrm{Xa}$ are also currently in development. In the $\mathrm{Xa} \mathrm{Neu-}$ tralization for Atherosclerotic Disease Understanding (XaNADU) phase II trial, the safety of the direct factor $\mathrm{Xa}$ inhibitor DX-9065a was assessed in patients undergoing elective, native-vessel PCI. ${ }^{30}$ During the PCI procedure, patients received either one of three dosages of DX-9065a or the standard regimen of UFH. However, use of the lowest dose regimen was halted abruptly after a serious thrombotic incident in the seventh patient to receive this dose. Procedural complications were also commonly reported in patients receiving the other DX-9065a dosages. Side branch closure after stent implantation was reported in three $(2.1 \%) \mathrm{pa}^{-}$ tients in stage 1 , and one (2.2\%) stage 4 patient suffered subacute stent thrombosis requiring urgent revascularization $\sim 12$ hours after PCI. Of the patients receiving $\mathrm{UFH}$, no procedural complications were reported. Although this study concluded that the use of DX9065a during PCI merited further investigation, the development of this particular factor $\mathrm{Xa}$ inhibitor was not continued.

More recently, another direct factor Xa inhibitor, otamixaban, was assessed in a double-blind, doubledummy, dose-ranging trial (SEPIA-PCI) in patients undergoing nonurgent $\mathrm{PCI}^{31}$ In this trial, patients were randomized to receive one of five otamixaban drug regimens or UFH with or without concomitant use of GP IIb/IIIa inhibitors. The highest dose of otamixaban reduced the plasma concentration of $\mathrm{F} 1+2$ prothrombin fragments significantly more than did UFH without causing increased bleeding. Only two serious cases of catheter-related thrombosis were reported during this trial, both occurring in patients receiving the lowest dose of otamixaban. No coronary artery or catheter-related thrombi in patients receiving alternative otamixaban doses or UFH during this study were considered to be serious. Further studies are required to evaluate the safety and efficacy of otamixaban for this indication.

Recently, oral antithrombin agents such as dabigatran and factor $\mathrm{Xa}$ inhibitors such apixaban and rivaroxiban were developed for the long-term management of ACS. Though their role in the management of PCI may be limited, these drugs in modified formulations can be used in PCI. Additionally, combined use of anti-Xa and anti-IIa drugs has also emerged in PCI. The coming years will witness major changes in anticoagulation therapy; however, the heparins will continue to play an important role in this area. 


\section{ANTIPLATELET DRUGS}

The introduction of novel antiplatelet drugs has added a new dimension to the management of arterial thrombosis; in particular, thrombotic stroke. The availability of specific antagonists of the ADP receptor (e.g., ticlopidine) has provided a new approach for several cardiovascular and cerebrovascular indications. The second-generation ADP receptor-blocking agents (e.g., clopidogrel) underwent extensive clinical trials to test their therapeutic efficacy in combined cardiovascular and cerebrovascular end points. ${ }^{32}$ The comparative results reported in several clinical trials have favored clopidogrel. ${ }^{33}$ The data on clopidogrel alone in various indications is rather limited. Actual clinical trials, where clopidogrel is compared with aspirin and other drugs, are therefore needed to determine the relative efficacy of this agent. In most studies performed to date, on-board aspirin has been used with clopidogrel. Clopidogrel has also been proved to be very important in preventing stent thrombosis. ${ }^{34}$

The newly developed antithrombin, anti-Xa, ADP receptor antagonists and recombinant antithrombotic drugs have only undergone limited and qualified clinical trials. Most of the data obtained from these studies is obtained through industry-sponsored studies. Thus, there is a need for unbiased and objective clinical results, which can only be obtained through postmarketing surveillance. This will require the unqualified regulatory approval of some of the newer drugs for multiple indications in which the conventional drugs have been used for a long time. All drugs exhibit some sort of safety problems. Because of the defined and monotherapeutic nature of the newer drugs, it has been projected that these drugs may have less toxicity and adverse reactions. However, even at this early stage, several safety issues with the newer drugs have already been identified. Table 3 lists some of the reported adverse reactions with the newer anticoagulant and antithrombotic drugs. Bleeding has been one of the most commonly observed

Table 3 Safety of Newer Drugs?

\begin{tabular}{lc}
\hline Drugs & Observed Concerns \\
\hline Hirudins & $\begin{array}{c}\text { Bleeding, antibodies, } \\
\text { accumulation } \\
\text { Granulocytopenia, TTP, } \\
\text { bleeding, free radical } \\
\text { formation (!) }\end{array}$ \\
Oral thrombin inhibitors & $\begin{array}{c}\text { Liver enzyme elevation, } \\
\text { bleeding } \\
\text { Anti-Xa drugs }\end{array}$ \\
r-TFPI & $\begin{array}{c}\text { Hemodynamic compromise, } \\
\text { drug interactions }\end{array}$ \\
Recombinant & Bleeding, antibodies \\
antithrombotic proteins & Antibodies, bioavailability \\
\hline
\end{tabular}

ADP, adenosine diphosphate; TTP, thrombotic thrombocytopenic purpura; r-TFPI, recombinant tissue factor pathway inhibitor. problems with these drugs. With the stipulation that fixed dosage may be applicable in all patients, bleeding risks may be higher with the newer drugs, as has been the reported case with fondaparinux. ${ }^{35}$ Thrombocytopenia, granulocytopenia, and immune thrombocytopenic purpura (ITP)-like syndrome have been reported with the ADP receptor inhibitors such as ticlopidine and clopidogrel. The use of oral thrombin inhibitors has been reportedly associated with liver enzyme elevation. The anti-Xa inhibitors have been associated with hemodynamic compromise and other side effects, and the recombinant antithrombin proteins have been associated with antibody generation.

It is now widely believed that the days for the classic anticoagulants are numbered and that in the foreseeable future these drugs may not exist. However, this is not the case when one reads the recommendations of the American College of Chest Physicians (ACCP) and the approval labels for the drugs. ${ }^{36}$ Considering the results of several new clinical trials, the ACCP and the International Union of Angiology consensus conferences on antithrombotic therapy have included definitive recommendations on the clinical effectiveness of the classic drugs in both arterial and venous diseases. In addition, these recommendations include specific guidelines on additional indications where these drugs will be useful. Thus, it is very likely that heparin, warfarin, and aspirin will continue to be important drugs in hematologic and oncologic disorders for some time. ${ }^{1,2,37}$

\section{CONVENTIONAL ANTICOAGULANTS}

When the classic anticoagulants are described in recent publications, they are often labeled as "bad" drugs with many adverse effects. In fact, the classic anticoagulants may not have any more adverse effects than do the new drugs. Needless to say, all pharmacologic agents have their limitations. Heparin, aspirin, and warfarin certainly have issues, some of which have already been addressed and improvements made.

The development of LMWHs is an example of the optimized use of a pharmacologic agent. Their use has nearly eliminated the risk of HIT, and these drugs have achieved standard of care status for many venous and arterial thrombosis indications. LMWHs have gradually replaced heparin in subcutaneous indications and are currently being examined for their effectiveness as surgical and interventional anticoagulation. Improved monitoring and dosage optimization are currently being pursued. Another example is the SPORTIF trial in which the oral anticoagulant warfarin was found to be essentially equivalent to the new oral antithrombin agent ximelagatran, without risk of significant bleeding. ${ }^{38}$ Moreover, warfarin use was not associated with elevation of liver enzymes. ${ }^{39}$ 
Despite the reported problem of HIT, heparin has remained the drug of choice for surgical anticoagulation. This is due to the high bleeding risk associated with the new antithrombin agents when used at higher doses coupled with the lack of antagonists. To the contrary, heparin has a well-tolerated antagonist, protamine, and the heparin-protamine combination has been used with much success for many years. Therefore, currently, UFH is the only reliable anticoagulant that can be used in surgical and interventional indications.

It is noteworthy to state that thrombosis is a polycomponent syndrome, which optimally requires a multiple target therapeutic approach. However, with the advanced understanding of the molecular and vascular biology of thrombotic disorders, only monotherapeutic drugs that have a single target of action have been developed. These monotherapeutic agents such as fondaparinux, dabigatran, and rivaroxiban are molecularly and functionally defined. Their applications have been validated in well-designed, sponsored clinical trials for specific indications. But, like the classic drugs, these new drugs were also found to have adverse effects. Bleeding and lack of dose response as well as monitoring and antidotes for overdosage remain problematic issues.

Further and not insignificantly, the net total sales of the three classic drugs is less than $\$ 3$ billion. The projected sales figures for the new alternate drugs is in the neighborhood of $\$ 15$ billion. In this age of cost constraints, can we afford the increased pricing of newer drugs?

Conventional drugs such as heparin, oral anticoagulants, and aspirin will remain the gold standards despite their known drawbacks. They require further optimization but can still currently be used for various indications in a cost-effective manner. The newer drugs may, however, provide alternatives that in the next few years could lead to improved cost-compliant treatments. The actions of the non-anticoagulant drugs such as the cholesterol-lowering agents (statins), specific inhibitors of cyclooxygenase, drugs capable of donating nitric oxide or upregulating its mediators, and drugs modulating endothelial function will also impact the combination therapy of thrombotic and cardiovascular diseases.

The branded LMWHs are now challenged with the insurgence of the generic, apparently equivalent products. At the present time, there are no clear guidelines for the acceptance of the generic LMWHs. The available guidelines are insufficient to address the complex issues related to the functional and structural characteristics of these drugs. Many countries around the world have already allowed generic versions of enoxaparin, dalteparin, and fraxiparin. It is important to track the clinical performance of these generic products and to recognize any potential differences between the generic and the innovative product. Such data may be helpful for the proper validation and potential acceptance of generic LMWHs. Warfarin has remained the sole oral anticoagulant for the extended management for thrombotic conditions. The generic versions of warfarin have been successfully introduced throughout the world including the United States. The use of INR for the monitoring of this oral anticoagulant has greatly facilitated harmonization in the dosing and has helped in eliminating reagent-based variations. Additionally, point-of-care testing devices and self-monitoring programs with guided dose adjustments have resolved many problems associated with monitoring.

\section{NEW ANTICOAGULANTS AND WARFARIN}

Initial attempts to develop an orally bioavailable product to replace warfarin have so far failed. The development of orally bioavailable thrombin inhibitors has also been initially targeted. Ximelagatran underwent extensive clinical trials in various indications, such as DVT prophylaxis in orthopedic surgery, atrial fibrillation, and secondary prevention of thromboembolism, with the obvious hope that it would replace warfarin for all indications. Its use was associated with elevation of liver enzymes in up to 8 to $10 \%$ of patients and a greater frequency of ACS complications in patients treated for the prophylaxis of DVT after orthopedic surgery. Subsequently, the FDA rejected the approval of ximelagatran for the triple indications. All of the orally bioavailable direct thrombin and $\mathrm{Xa}$ inhibitors, such as ximelagatran, dabigatran, rivaroxiban, and apixiban, represent synthetic organomimetic compounds with certain structural similarities to ximelagatran and are likely to be metabolized by the liver (Fig. 4). Moreover, all of these compounds pass through the placental barrier and have a potential to rebound. It is likely that regulatory bodies will require additional studies on the safety of these drugs. Although there is an apparent need to replace warfarin and related oral anticoagulants by more effective, predictable, and safer drugs, the FDA's concern is valid and not until these safety issues are resolved will the anti-Xa and anti-IIa drugs be clinically accepted.

There are several other oral thrombin inhibitors currently being developed by different companies (Fig. 5). Most of these represent synthetic heterocyclic compounds. It is conceivable that all of these agents may have a similar performance profile as discussed above. An additional concern, however, is the fact that thrombin exhibits several regulatory functions. Of major importance is the thrombin-thrombomodulin pathway. All thrombin inhibitors that produce a sustained inhibition of thrombin are expected to produce an inhibition of this pathway leading to varying degrees of hemostatic regulatory compromise. Oral thrombin inhibitors may exhibit a class effect unless there are major modifications in the molecular design of these agents. 


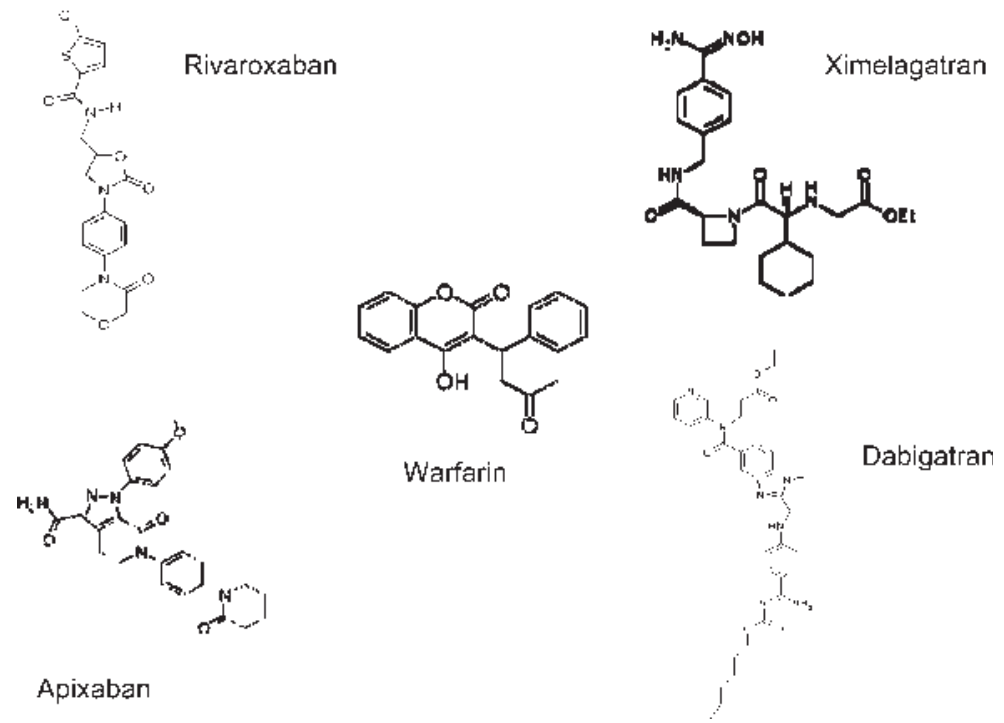

Figure 4 The structures of various synthetic anti-Xa and anti-lla agents are comparable and are mainly composed of nitrogencontaining heterocyclic structures. Ximelagatran and dabigatran are prodrugs that require metabolic activation to mediate their antithrombotic effects. Rivaroxaban and apixaban are direct-acting anti-Xa agents. Warfarin has structural similarities to these anti-Xa and anti-Ila agents as it has aromatic residues. All agents are expected to be metabolized by liver enzymes and pass through the placental barrier due to their small size. The scaffold structure of the newly developed anti-Xa and anti-lla drugs is similar, and these may exhibit similar pharmacologic and toxic profiles.

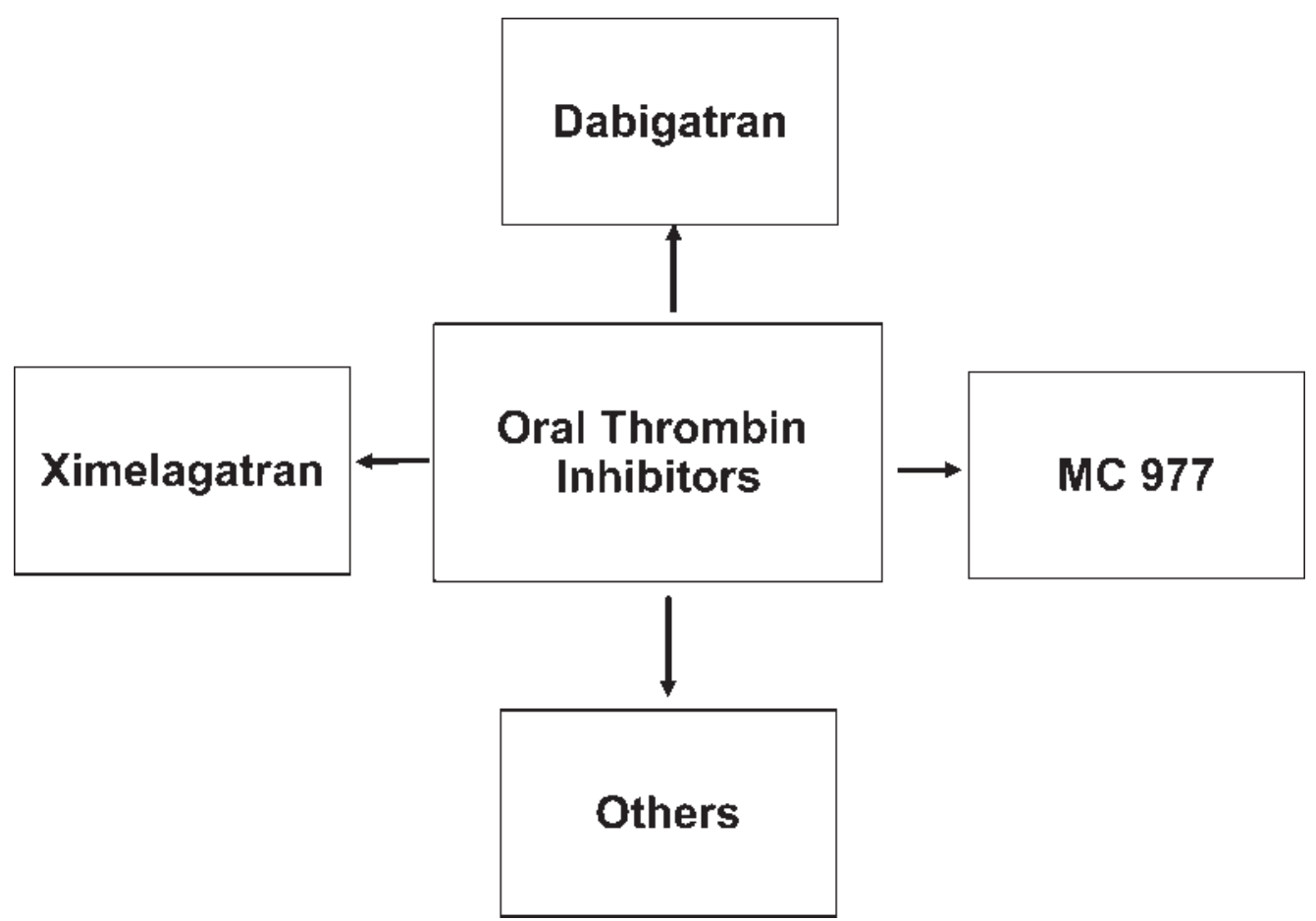

Figure 5 Several synthetic antithrombin agents are currently in various stages of clinical development, whereas many others are in different stages of preclinical development. The development of ximelagatran (AstraZeneca, Wilmington, DE) has been stopped because of its toxic profile. Dabigatran (Boehringer Ingelheim, Ridgefield, CT) is in advanced clinical stages for several indications. MC-977 (Mitsubishi, Tokyo, Japan) is in early clinical development for postsurgical venous thrombosis. The therapeutic spectrum of these agents is also similar, and they all represent specific organic agents with molecular weight $<1000 \mathrm{Da}$. 
Table 4 Newer Oral Anti-Xa and Anti-lla Agents and Their Comparison with Warfarin

\begin{tabular}{lllll}
\hline Agent & Class & $\begin{array}{l}\text { Half-life } \\
\text { (h) }\end{array}$ & $\begin{array}{l}\text { Renal } \\
\text { Clearance } \\
\text { (mL/min) }\end{array}$ & $\begin{array}{l}\text { Placental } \\
\text { Passage }\end{array}$ \\
\hline Rivaroxaban & Anti-Xa & $10-12$ & 70 & Yes \\
$\begin{array}{l}\text { Apixaban } \\
\text { Dabigatran }\end{array}$ & Anti-Xa & $10-15$ & 30 & Yes \\
Warfarin & $\begin{array}{l}\text { Dysfunctional } \\
\text { forms of II, VII, }\end{array}$ & $30-50$ & 40 & Yes \\
& IX, and X & & & \\
\hline
\end{tabular}

Table 4 shows a comparison of the half-life, renal clearance, and the potential for placental passage for various anti-Xa and anti-IIa agents with warfarin. All of these drugs exhibit similar characteristics to warfarin and are cleared through both renal and hepatic mechanisms. All are capable of passing the placental passage. Therefore, the toxicity associated with warfarin may also be observed with these drugs.

On the other hand, the direct thrombin inhibitors (DTIs) such as argatroban, lepirudin, and bivalirudin have been extremely useful in the acute and short-term management of heparin-compromised patients who require treatment or anticoagulation for interventional procedures. The 7th ACCP guidelines have provided specific recommendations for the use of DTIs in heparin-compromised patients. However, the data are somewhat limited. At comparable anticoagulation levels (e.g., using activated partial thromboplastin time $[\mathrm{aPTT}]$ or activated clotting time $[\mathrm{ACT}]$ activity), the pharmacologic profiles of the different DTIs are distinct from each another. These drugs also exhibit different degrees of interactions with other drugs. This should be taken into account in their development.

The parenteral DTIs are currently administered by intravenous bolus or infusion. However, subcutaneous formulations for these agents will soon become available. As the patents of some DTIs are near expiration or have expired, generic versions of these DTIs will become available. Because these drugs are synthetic or biosynthetic (recombinant), applicable guidelines developed for specific groups must apply. Currently, there are no guidelines available. Furthermore, the pharmacologic differences among these drugs are not addressed at this time. It is expected that newer trials may provide some insight on the differentiation of these agents. ${ }^{37}$

A comparison of warfarin and enoxaparin with the newly developed anti-Xa and anti-IIa agents is shown in Table 5. The clinical indications for these agents are similar; however, warfarin has a broader usage. Warfarin was approved in 1954 for arterial and venous thrombosis. The first LMWH, enoxaparin, was approved in the United States in 1983 for venous thromboembolism (VTE) and later ACS. In 2004, fondaparinux was approved for postsurgical VTE management. Now it is being considered for ACS; however, there are several safety issues. Dabigatran is slated to be approved in 2010, whereas rivaroxiban was initially targeted for approval in 2009. Because of the liver enzyme issues and thrombosis rebound, the regulatory approval of these drugs may be delayed.

\section{CONCLUSION}

The progress in the development of newer antithrombotic and anticoagulant drugs has been remarkable in the past decade. However, the focus of these developments has been toward the introduction of monotherapeutic agents with known targets. For this reason, antithrombin, anti-factor $\mathrm{Xa}$, anti-tissue factor, and specific inhibitors toward active coagulation enzymes such as factors VIIa, IXa, XIIa, and XIIIa are also being developed. Many of these drugs have undergone phase I and phase II clinical trials but despite high expectations have failed to provide expected outcomes. As thrombosis is a polypathologic process, it requires a polypharmacologic agent for management. Single targeting of thrombotic and cardiovascular diseases may not provide desirable outcomes. Thus, drugs with a polytherapeutic profile such as the LMWHs and combination regimens may be more effective in the management of thrombosis.

The withdrawal of rofecoxib by Merck (Vioxx; Merck, Rahway, NJ) is a case in point for the need of polytargeting approaches. Aspirin and nonsteroidal antiinflammatory drugs, besides being anti-inflammatory, also have specific effects by regulating COX-1 and COX-2. An intricate balance is needed between these enzymes to regulate physiologic functions. To manage inflammation, COX-2 inhibition was targeted and COX-1 processes were untouched. This distorted the

Table 5 A Comparison of Various Anticoagulant Drugs

\begin{tabular}{lllll}
\hline Drug & Trade Name & Company & FDA-Approved Indication & $\begin{array}{c}\text { Date of FDA } \\
\text { Approval }\end{array}$ \\
\hline Warfarin & Coumadin & Bristol Myers-Squibb, New York, NY & Arterial and venous thrombosis & 1954 \\
Enoxaparin & Lovenox & Sanofi Aventis, Bridgewater, NJ & VTE/ACS & 1993 \\
Fondaparinux & Arixtra & Glaxo-Smith Kline, Philadelphia, PA & VTE/ACS & 2004 \\
Dabigatran & Rendix & Boehringer Ingelheim, Ridgefield, CT & VTE/arterial fibrillation & 2010 \\
Rivaroxaban & Xarelto & Bayer Healthcare, Leverkusen, Germany & VTE/arterial fibrillation & 2009 \\
\hline
\end{tabular}


natural balance, resulting in the reported cardiovascular complications in patients treated with rofecoxib. Similarly, in the case of monotargeting anti-factor $\mathrm{Xa}$, antithrombin, and anti-tissue factor drugs, the polypharmacologic effects of heparins and oral anticoagulants are compromised. This may be the reason why DTIs exhibit rebound and other observed vascular complications.

The events leading to the rejection of ximelagatran by the FDA advisory committee and the voluntary withdrawal of rofecoxib by Merck are a clear testimony to the problems related to antithrombotic drug development. This should alert the pharmaceutical industry to a greater need for carrying out more extensive preclinical pharmacologic studies. This will help identify major problems at the initial stages of the development of new antithrombotic drugs and will reduce unforeseen patient compromise at the later stages of development. At the same time, besides evaluating the major clinical end points, substudies within clinical trials can be designed to monitor endogenous physiologic/hemostatic effects of new drugs, as well as drug combinations, and to project therapeutic indices. This was apparently not done with rofecoxib and ximelagatran. As a rule, the pharmaceutical industry has resisted such opportunities because of fiscal and logistic constraints. However, major catastrophic and financial losses can be avoided by properly designing a drug development program.

In a sense, heparins and warfarin both represent polypharmacologic agents. These drugs produce their effects via multiple mechanisms besides inhibiting thrombin and $\mathrm{Xa}$, which are the primary target of the anti-Xa and anti-IIa drugs. Table 6 shows that LMWHs produce their effects by multiple mechanisms, whereas the direct anti-Xa and anti-IIa agents have a much narrower spectrum. Therefore, it is unlikely that the newly developed synthetic anti-Xa and anti-IIa drugs will produce similar effects as those achieved by heparin and warfarin.

Table 6 Polypharmacology of Heparins Compared with Synthetic Organomimetic Anti-Xa and Anti-lla Agents

\begin{tabular}{llll}
\hline Attributes & LMWHs & Anti-Ila & Anti-Xa \\
\hline Inhibition of thrombin & + & +++ & \pm \\
Inhibition of Xa & ++ & \pm & +++ \\
Inhibition of thrombin & +++ & + & +++ \\
$\quad$ generation & & & - \\
Release of TFPI & ++ & - & - \\
Activation of fibrinolysis & + & \pm & - \\
Endothelial effects & ++ & - & + \\
Modulation of platelet & + & + & - \\
$\quad$ function & & - & \\
Growth factor modulation & ++ & &
\end{tabular}

-, not present; \pm , may be present; +, present; ++, moderately present; +++ , strongly present.

LMWHs, low molecular weight heparins.
Currently, rivaroxiban and apixiban represent the two oral anticoagulants that are in advanced clinical development for the management of venous thrombosis and atrial fibrillation. In addition, these agents are also aggressively developed for arterial thrombosis and venous thrombosis in medical patients. The clinical results obtained with fondaparinux validated the concept that sole anti-Xa agents are useful in the management of thrombosis, whereas the results with antithrombin agents such as lepirudin and ximelagatran validated the use of sole antithrombin agents in the management of thrombosis. Dabigatran represents the lead antithrombin agent, which is developed for the same indications as the oral anti-Xa agents, such as apixiban and rivaroxiban. One of the most commonly asked questions is related to the comparison of the anti-Xa and anti-IIa agents, as these agents represent different targets. Remarkably, in the clinical trials, both classes of drugs have performed similarly. Although the safety issues such as bleeding with anti-Xa agents are reportedly less concerning than with the anti-IIa agents, thrombosis rebound and liver enzyme elevation has been reported with this class of drugs. The long-term use of thrombin inhibitors may inhibit the regulatory functions of thrombin such as the activation of protein $\mathrm{C}$ and thrombin activatable fibrinolytic inhibitor (TAFI). Also, hemostatic effects, including thrombin receptor activation in platelets and activation of factors $\mathrm{V}$ and XIII, may be impaired. This may be the reason for enhanced bleeding with antithrombin agents. On the other hand, the Xa inhibitors may exhibit reduced efficacy in patients with preformed thrombin, which can only be inhibited using antithrombin agents. For this reason, newer agents containing both anti-Xa and anti-IIa activities are being developed. It is too early to comment on the relative safety and efficacy of these two classes of drugs. However, the parenteral use of antithrombin agents has been clinically validated. It is unlikely that factor $\mathrm{Xa}$ agents will have similar performance as the parenteral antithrombin agents.

It is likely that the newly developed monotherapeutic agents may also exhibit additional unknown effects, which have not been completely explored at this time. Moreover, these agents represent organomimetic drugs, which can be metabolized into active agents whose pharmacologic profile is unknown at this time. All of the newly developed anti-Xa and anti-IIa agents represent nitrogen-containing heterocyclic compounds. Some of these may contribute to the regulation of nitric oxide and produce hemodynamic modulation. Similarly, the new antiplatelet agents may also transform into active and inactive metabolites with differential pharmacologic effects. The behavior of these drugs may differ in different populations, and generic polymorphism may strongly influence their therapeutic profile. Thus, unlike heparin, warfarin, and aspirin where the 
safety and efficacy profiles are now well-known, the same level of information on the newer drugs is not available and will take some time to generate for the optimization process.

Finally, the regulatory bodies will have to assume a greater responsibility in directing the pharmaceutical industry toward objective approaches to evaluate a new drug in a given indication. This will require the creation of dedicated divisions within the FDA and the EMEA with expertise in hemostasis and thrombosis. At the same time, closer interactions between the different divisions within the FDA should be encouraged. This will facilitate the recognition of specific issues related to the development of new antithrombotic and anticoagulant drugs, which represent a diverse group of therapeutic agents with both structural and biological heterogeneity. Moreover, a stronger scrutiny of drug development programs with more stringent monitoring of the different phases of clinical development by the FDA is warranted to ensure unbiased clinical trial conduct.

\section{REFERENCES}

1. Fareed J, Hoppensteadt DA, Bick RL. Management of thrombotic and cardiovascular disorders in the new millennium. Clin Appl Thromb Hemost 2003;9:101-108

2. Fareed J, Hoppensteadt DA. The management of thrombotic and cardiovascular disorders in the 21st century. In: Sasahara AA, Loscalzo J, eds. New Therapeutic Agents in Thrombosis and Thrombolysis. 2nd ed. New York, NY: Marcel Dekker; 2002:687-693

3. Eikelboom JW, Weitz JI. A replacement for warfarin: the search continues. Circulation 2007;116:131-133

4. Bates SM, Weitz JI. The status of new anticoagulants. Br J Haematol 2006;134:3-19

5. Laux V, Hinder M. Rationale for direct factor $\mathrm{Xa}$ inhibitors in acute coronary syndromes. In: Kipshidze N, Fareed J, Moses JW, Serruys PW, eds. Textbook of International Cardiovascular Pharmacology. Andover, UK: Informa Healthcare; 2007: 119-126

6. Leong W, Hoppensteadt DA. Generic forms of low molecular weight heparins: some practical considerations. Clin Appl Thromb Hemost 2003;9:293-297

7. Marmur JD, Anand SX, Bagga RS, et al. The activated clotting time can be used to monitor the low molecular weight heparin dalteparin after intravenous administration. J Am Coll Cardiol 2003;41:394-402

8. Fox KA. Implications of the organization to assess strategies for ischemic syndromes-2 (OASIS-2) study and the results in the context of other trials. Am J Cardiol 1999;84:26M$31 \mathrm{M}$

9. Lee AY, Levine MN, Baker RI, et al. Low-molecular-weight heparin versus a coumarin for the prevention of recurrent venous thromboembolism in patients with cancer. N Engl J Med 2003;349:146-153

10. Linkins LA, Weitz JI. Pharmacology and clinical potential of direct thrombin inhibitors. Curr Pharm Des 2005;11:38773884
11. Antman EM. Hirudin in acute myocardial infarction. Thrombolysis and thrombin inhibitors in myocardial infarction (TIMI) 9B trial. Circulation 1996;94:911-921

12. Cho L, Bhatt DL, Marso SP, et al. An invasive strategy is associated with decreased mortality in patients with acute coronary syndrome and non-ST elevation myocardial infarction: GUSTO IIb trial. Am J Med 2003;114:106111

13. Eriksson BI, Ekman S, Lindbratt S, et al. Prevention of thromboembolism with use of recombinant hirudin: results of a double blind, multicenter trial comparing the efficacy of desirudin (Revasc) with that of unfractionated heparin in patients having a total hip replacement. J Bone Joint Surg Am 1997;79:326-333

14. Eriksson BI, Wille-Jorgensen $\mathrm{P}$, Kalebo $\mathrm{P}$, et al. a comparison of recombinant hirudin with low-molecular-weight heparin to prevent thromboembolic complications after total hip replacement. N Engl J Med 1997;337:1329-1335

15. Lincoff AM, Bittl JA, Harrington RAet al. for the REPLACE-2 Investigators. Bivalirudin and provisional glycoprotein IIb/IIIa blockade compared with heparin and planned glycoprotein IIb/IIIa blockade during percutaneous coronary intervention. REPLACE-2 randomized trial. JAMA 2003;289:853-863

16. Stone GW, McLaurin BT, Cox DAet al. for the ACUITY investigators. Bivalirudin for patients with acute coronary syndromes. N Engl J Med 2006;355:2203-2216

17. Stone GW, Bertrand ME, Moses JW, et al. Routine upstream initiation vs. deferred selective use of glycoprotein IIb/IIIa inhibitors in acute coronary syndromes: the ACUITY Timing trial. JAMA 2007;297:591-602

18. Stone GW, White HD, Ohman EM, et al. Bivalirudin in patients with acute coronary syndromes undergoing percutaneous coronary intervention: a subgroup analysis from the Acute Catheterization and Urgent Intervention Triage strategy (ACUITY) trial. Lancet 2007;369:907-919

19. Eichler P, Lubenow N, Strobel U, Greinacher A. Antibodies against lepirudin are polyspecifc and recognize epitopes on bivalirudin. Blood 2004;103:613-616

20. Lewis BE, Wallis DE, Berkowitz SD, et al. Argatroban anticoagulant therapy in patients with heparin-induced thrombocytopenia. Circulation 2001;103:1838-1843

21. Lewis BE, Wallis DE, Leya F, et al. Argatroban anticoagulation in patients with heparin-induced thrombocytopenia. Arch Intern Med 2003;163:1849-1856

22. Dahl OE, Eriksson BI, Agnelli G, et al. Postoperative melagatran/ximelagatran for the prevention of venous thromboembolism following major elective orthopedic surgery: effects of timing of finishing factors for thromboembolism and bleeding complications on efficacy and safety. Clin Drug Investig 2005;25:65-77

23. Kalus JS, Caron MF. Novel uses for current and future direct thrombin inhibitors; focus on ximelagatran and bivalirudin. Expert Opin Investig Drugs 2004;13:465-477

24. Turpie AG, Fisher WD, Bauer KA, et al. BAY 59-7939: an oral, direct factor $\mathrm{Xa}$ inhibitor for the prevention of venous thromboembolism in patients after total knee replacement. A phase II dose-ranging study. J Thromb Haemost 2005;3: 2479-2486

25. Erikkson BI, Borris L, Dahl O, et al. Oral, direct factor Xa inhibition with Bay 59-7939 for the prevention of venous thromboembolism after total hip replacement. J Thromb Haemost 2006;4:121-128 
26. Yusuf S, Mehta SR, Chrolavicius S, et al. Effects of fondaparinux on mortality and reinfarction in patients with acute ST-segment elevation myocardial infarction: the OASIS-6 randomized trial. JAMA 2006;295:1519-1530

27. Saiah E, Soares C. Small molecule coagulation inhibitors in the clinic. Curr Trends Med Chem 2005;5:1677-1695

28. Aherns I, Smith BK, Bode C, Peter K. Direct thrombin inhibition with bivalirudin as an antithrombotic strategy in general and interventional cardiology. Expert Opin Drug Metab Toxicol 2007;3:609-620

29. Lewis BE, Matthai WH Jr, Cohen M, et al. Argatroban anticoagulation during percutaneous coronary intervention in patients with heparin-induced- thrombocytopenia. Catheter Cardiovasc Interv 2002;57:177-184

30. Alexander JH, Yang H, Becker RC, et al. First experience with direct, selective factor $\mathrm{Xa}$ inhibition in patients with non-ST-elevation acute coronary syndromes: results of the Xa NADV-ACS trial. J Thromb Haemost 2005;3: 439-447

31. Cohen M, Bhatt DL, Alexander JH, et al. Randomized, double-blind, dose-ranging study of otamixaban, a novel, parenteral, short-acting direct factor $\mathrm{Xa}$ inhibitor, in percutaneous coronary intervention: the SEPIA-PCI trial. Circulation 2007;115:2642-2651

32. CAPRIE Steering Committee. A randomized, blinded, trial of clopidogrel versus aspirin in patients at risk of ischemic events (CAPRIE). Lancet 1996;348:1329-1339

33. Smith SC, Feldman TE, Hirschfeld JW, Jacobs AK. American College of Cardiology/American Heart Associa- tion Task Force on practice guidelines. ACC/AHA/SCA 2005. Circulation 2006;113:e166-e186

34. Bhatt DL, Bertrand ME, Berger PB. Meta-analysis of randomized and registry comparison of ticlopidine with clopidogrel after stenting. J Am Coll Cardiol 2002;39:9-14

35. Bauer KA, Eriksson BI, Lassen MR, Turpie AG. Fondaparinux compared with enoxaparin for the prevention of venous thromboembolism after elective major knee surgery. N Engl J Med 2001;345:1305-1313

36. Hirsh J, Guyatt GH, Albers GW, Schunemann HJ. The 7th ACCP Conference on Antithrombotic and Thrombolytic Therapy: evidence-based guidelines. Chest 2004;126:163S$703 \mathrm{~S}$

37. Fareed J. Anticoagulant management of patients undergoing interventional procedures. In: Kipshidze N, Fareed J, Moses JW, Serruys PW, eds. Textbook of International Cardiovascular Pharmacology. Andover, UK: Informa Healthcare; 2007:613-619

38. Flaker GC, Gruber M, Connolly SJ, et al. SPORTIF Investigators. Risks and benefits of combining aspirin with anticoagulant therapy in patients with atrial fibrillation: an exploratory analysis of stroke prevention using an oral thrombin inhibitor in atrial fibrillation (SPORTIF) trials. Am Heart J 2006;152:967-973

39. Peterson P, Grind M, Adler J. Ximelagatran versus warfarin for stroke prevention in patients with nonvalvular atrial fibrillation. SPORTIF II: a dose guiding tolerability and safety study. J Am Coll Cardiol 2003;41: 1445-1451 